\title{
INFORMATION QUALITY EVALUATION FOR GRID INFORMATION SERVICES
}

\author{
Wei Xing, Oscar Corcho, Carole Goble \\ School of Computer Science \\ University of Manchester \\ United Kingdom \\ wxing@cs.man.ac.uk \\ ocorcho@cs.man.ac.uk \\ carole@cs.man.ac.uk \\ Marios Dikaiakos \\ Department of Computer Science \\ University of Cyprus, Cyprus \\ mdd@cs.ucy.ac.cy
}

\begin{abstract}
The quality of the information provided by information services deployed in the EGEE production testbed differs from one system to another. Under the same conditions, the answers provided for the same query by different information services can be different. Developers of these services and of other services that are based on them must be aware of this fact and understand the capabilities and limitations of each information service in order to make appropriate decisions about which and how to use a specific information service. This paper proposes an evaluation framework for these information services and uses it to evaluate two deployed information services (BDII and RGMA) and one prototype that is under development (ActOn).
\end{abstract}

Keywords: Grid, Grid Information Service, Information Quality, Evaluation 


\section{Introduction and Motivation}

Information Services are regarded as a vital component of the Grid infrastructure. They address the challenging problems of the discovery and continuous monitoring of all types of Grid resources, including services, hardware, software and other entities. The quality of information provided by information systems affects the performance and the behaviour of other dependent Grid services. For instance, a Grid meta-scheduling service will not work optimally if the quality of the information used for decision making is poor; a Grid Resource Broker depends heavily on the quality of the information about Grid resources provided by the information services that it uses; etc.

There is currently little work done on the evaluation of information quality of Grid information services. Most evaluation studies focus on performance measurement [1-2], such as evaluating scalability, overload, query response time, etc. Such measurements are based on the assumption that information quality is equal for different information services. However, this assumption does not hold in reality, since each information system has different mechanisms for collecting and processing information, and adopts different information models for storage and querying. This is something covered in our experiments, which show that even for a simple query different systems provide extremely different results. For example, we submitted the query "find me Computing Elements which support the Biomed Virtual Organisation" simultaneously to the two EGEE default information services, BDII and RGMA, and they had different results: BDII returned 151 and RGMA returned only 30. Independently of the reasons for such variable results, the main outcome from this simple test is that information quality of currently-deployed Grid information services has to be considered carefully.

The work described in this paper has several objectives. First, we want to obtain a fair systematic approach to measure information quality of different Grid information services, so that we can compare them and provide guidelines related to the circumstances in which each of them can be used. The main challenge here is related to the fact that different Grid information services have different information models to represent the same type of Grid resources: some of them use LDAP to represent that information and others use relational models, and the information that they store about each resource may also differ. Unlike information quality evaluation in other domains (such as Web search, where precision and recall measurements can be obtained by counting numbers of documents), the information objects in our evaluation are heterogeneous, both in the information model used and in its access API, what makes it hard to compare the outputs. We have proposed the use of a common information model to allow comparisons between these outputs. We explain the details in Section 3.2.

Another challenge to be overcome is related to the differences in the querying capabilities and expressiveness supported by each service, what makes it difficult to design a good set of relevant experiments for the evaluation. Some services allow making complex queries that relate information from different domains (computing elements that support a specific virtual organisation and a specific software environment) and others just provide simple querying functionalities. In our approach we have proposed a set of representative queries that may be issued by other middleware services or applications that use these information services, and which have increasing levels of complexity.

Our second objective is to use the proposed approach for the information quality evaluation of two information services (BDII and RGMA) deployed in the EGEE Grid and one prototype that is under development (ActOn). We will analyse the results obtained from this evaluation and identify the reasons for obtaining such results. We think that these results can be used by developers working on these Grid information services, in order to improve them, and by developer of systems that are based on them.

The remaining of this paper is organised as follows. Section 2 describes briefly the information systems to be evaluated. Section 3 introduces our evaluation framework, including the design rationale, the experiments to be carried out, and the metrics to be used for the evaluation, together with details about how they are measured for each system. Section 4 describes the 


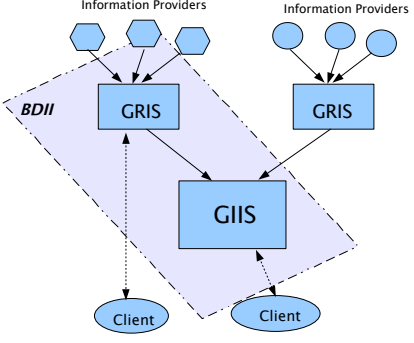

(a) BDII

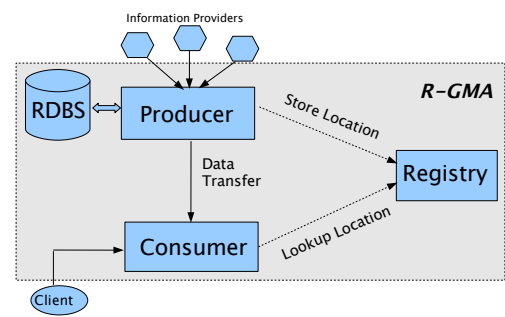

(b) RGMA

Figure 1. Overview of the BDII and RGMA architectures

results of the experiments carried out, and provides some conclusions related to these results. Finally, Section 5 reflects about the lessons learnt in the design of this evaluation framework and gives references to additional performance tests that we have carried out.

\section{Grid Information Services}

Currently, there are several well-known and widely-used Grid information services: Monitoring and Discovery System (MDS), Berkeley DB Information Index (BDII), and RGMA [3-5]. These services are deployed in most Grid systems, such as Europe Data Grid, Crossgrid, NASA Grid, and Open Science Grid [6-10], and widely used by Grid middleware and applications running on them. From these three services, we will select BDII and RGMA for our evaluation, since they are the default information services for the EGEE Grid. We do not include MDS in our evaluation because it is not deployed as the information service used for Computing Elements (CEs) and Sites in EGEE and would make difficult to perform the comparison. Besides, BDII is based on MDS, with the same information model (information representation and access), hence the general results regarding information quality and recommendations obtained for BDII could be easily extrapolated to MDS.

Besides these two services, we will evaluate the ActOn-based information service [11], an ontology-based information service recently developed by our group, which integrates information from both of these services. In fact, we started the development of this service when we realised that the quality of the information provided by the existing information services was not good enough, as we will show in Section 4.

In this section, we describe these three information services in detail. Table 3 summarises some of their main features, including their information model, information access protocol, and other aspects related to their architecture.

\subsection{Berkeley DB Information Index (BDII)}

BDII [4] is an improvement of MDS [3] , the information service component of the Globus platform. It uses the MDS information model and access API and caches information with the Berkeley DB. In its current version, MDS2.x, information about Grid resources is extracted by "information providers", which are software programs that collect and organise information from individual Grid entities, either by executing local operations or by contacting third-party information sources (e.g., the Network Weather Service, SNMP, etc.).

Extracted information is organised according to the LDAP (Lightweight Directory Access Protocol) data model, in LDIF format, and uploaded into LDAP-based servers of the Grid Resource Information Service (GRIS), as shown in Figure 1(a). GRIS servers can register themselves in the Grid Index Information Services (GIIS) in order to aggregate directories, using a soft-state registration protocol called Grid Registration Protocol (GRRP). 
An update process is used to populate LDAP-based servers. It consists in obtaining LDIF, either by doing an ldapsearch on LDAP URLs or by running a local script that generates LDIF. Then the LDIF is inserted into the LDAP database.

Currently, there are around 200 BDII servers (site BDII plus regional BDII) deployed in the EGEE production testbed.

\subsection{Relational Grid Monitoring Architecture (RGMA)}

RGMA [5] is a framework that combines monitoring and information services based on a relational model, which is implemented with XML. It has been built in the context of the EU DataGrid project and implements the Grid Monitoring Architecture (GMA) proposed by the Open Grid Forum.

As shown in figure 1(b), GMA models the information infrastructure of the Grid using three core types of components: (i) producers, which provide information; (ii) consumers, which request information; and (iii) a single registry, which mediates the communication between producers and consumers.

RGMA implements two additional properties over GMA. First, consumers and producers handle the registry in a transparent way; thus, anyone using RGMA to supply or receive information does not need to know about the registry. And second, all the information appears as one large relational database and can be queried as such (anyway, in the current implementation, the database is centralised). RGMA can be accessed using the RGMA API.

In the EGEE production testbed, there are 110 sites, for each of which there is a monitoring node (MON) that has an RGMA server. All of them use a centralised registry server located at lcgic01.gridpp.rl.ac.uk.

\subsection{Active Ontology (ActOn)-based information service}

ActOn [11] is an ontology-based information integration system that can be used to generate and maintain up-to-date information for a dynamic, large-scale distributed system, such as a Grid system. It has been developed by the Information Management Group at the University of Manchester. The ActOn architecture is comprised of a set of knowledge components, which represent knowledge from the application domain (e.g., the EGEE Grid) and from the information sources (e.g., RGMA and BDII servers); and software components, such as a metadata scheduler (MSch), an information source selector (ISS), a metadata cache (MC), and a set of information wrappers. Figure 2 shows how these components are interrelated and how they are related to the corresponding information sources where data is taken from.

Two distinct features of the ActOn system, when compared to other information integration systems, are: 1) it has an intermediate information source selection step that takes into account the current information needs and the state of the information services to be accessed;2) and it also includes a "metadata cache" that works with an update-on-demand policy, which avoids continuous update requests by aggregating only the metadata that is being queried.

The service that we will evaluate is a deployment of the ActOn system that uses BDII and RGMA as information sources, and a Grid Ontology [12-13] as its information model, and has been deployed in the EGEE certificate and production testbeds.

\section{An evaluation framework for information quality in Grid information services}

Information quality (IQ) can be defined as a measure of the value of the information provided by an information system to its users [14]. There are many characterisations of what quality means in this context (taking into account that quality is normally subjective and depends on the 


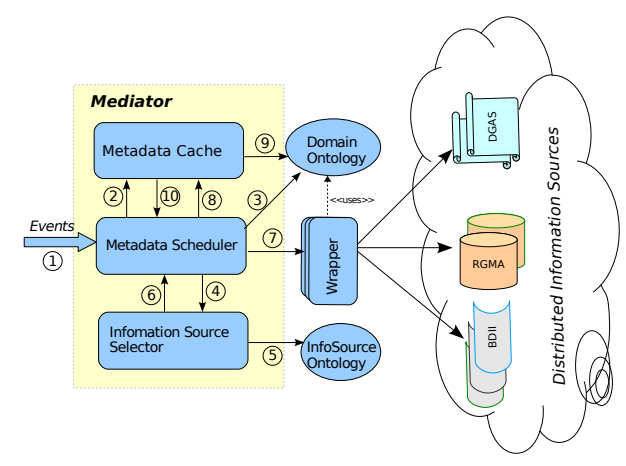

Figure 2. Overview of the Active Ontology architecture

\begin{tabular}{|c|c|c|c|c|c|c|c|}
\hline & Data Model & $\begin{array}{l}\text { Representation } \\
\text { language }\end{array}$ & $\begin{array}{l}\text { Query } \\
\text { Language }\end{array}$ & Architecture & Semantic-able & $\begin{array}{l}\text { Metadata } \\
\text { Cache }\end{array}$ & $\begin{array}{l}\text { Information } \\
\text { Access }\end{array}$ \\
\hline BDII & Tree & LDIF & LDAP & Centralized & No & Yes & Data Warehouse \\
\hline RGMA & Relational & XML & SQL & Distributed & No & No & On-demand \\
\hline $\begin{array}{l}\text { ActOn-based } \\
\text { Information service }\end{array}$ & Graph-based & RDF/RDFS/OWL & SPARQL & Centralized & Yes & Yes & $\begin{array}{l}\text { On-demand } \\
\text { +Data Warehouse }\end{array}$ \\
\hline
\end{tabular}

Figure 3. Features of the most common Grid information services

intended use of the information by users). The authors in [14] distinguish between intrinsic, contextual, representational and accessibility IQ, and define different factors to be considered for each of them (accuracy, objectivity, reputation, relevancy, etc.).

The authors in [15-16] propose to focus on seven of these characteristics, which are considered the most important ones, independently of their domain: completeness, accuracy, provenance, conformance to expectations, logical consistency and coherence, timeliness, and accessibility. In our framework we have selected three of these features, namely completeness, accuracy and conformance to expectations.

We are not worried about the provenance of information, since we know clearly which are the information sources that we use in each moment and which are the information providers responsible for that information. We are not worried either about accessibility, since we assume that the systems work within a Grid security infrastructure (e.g., GSI [17]), so that the information is accessible as long as the client has the corresponding rights to access it and knows the information model and API used by the corresponding information service.

With respect to the logical consistency and coherence and the timeliness of the information retrieved and aggregated from the information sources, these are features that will form part of our future evaluation work, and will be also considered in further developments of the ActOnbased information service. An example of why the first feature is important is the following: there are many cases where a computing element specifies that it gives support to MPI but does not comply with the requirements for running an MPI job, which are that it must be a CE server, must have an sshd service running on it, must have the libraries mpirun and libmpi.so in its file system, and must have at least two worker nodes. Information services like BDII or RGMA only store and provide the information that their information producers give them, without checking their consistency, hence they provide incorrect information due to this fact. As an example of the second feature, BDII normally updates the information that has been 
provided by its information sources every five or six minutes, what means that this information may be already inaccurate when a client requests it. Hence, having metadata about the lifetime and freshness of information in the information service is important.

In this section we describe our evaluation framework for information quality, including metrics to be used for the evaluation, the design rationale, and the experiments to be carried out, together with details about how the metrics are obtained for each system.

\subsection{Evaluation metrics}

To check the three criteria considered in our framework, we are interested in knowing whether all information services obtain the same results when answering the same query, given the same conditions in the EGEE testbed. We also want to check how many of those answers are correct and how many of the existing answers are actually retrieved. This also permits us to know whether the results provided by the services conform to the expectations of the users. To check this, we have selected two metrics, commonly used in information retrieval: precision and recall. Below we provide their definitions and the formulae used to calculate them:

Precision: The proportion of relevant information retrieved, out of all the information retrieved.

$$
\text { Precision }=\frac{(\text { relevant information }) \cap(\text { retrieved information })}{\text { retrieved information }}
$$

Recall: The proportion of relevant information that is retrieved, out of all the relevant information available.

$$
\text { Recall }=\frac{(\text { relevant information }) \cap(\text { retrieved information })}{\text { relevant information }}
$$

\subsection{Experiment setup and design}

We have designed a set of experiments for measuring the information quality criteria selected. Measurements are taken on a real Grid testbed, the EGEE production testbed, which at the time of the experiments, has gLite 3.0.1 installed as its middleware. The user interfaces used to access the EGEE Grid are the UI machines at the University of Manchester ${ }^{1}$, United Kingdom, and at the Institute of Physics of Belgrade ${ }^{2}$, Serbia.

To carry out the experiments and record their results, we have developed a set of Java-based client software and Unix shell scripts, available at the IST OntoGrid project CVS [18].

The key aspects upon which we compare different information services are: i) the information model that each information service adopts; and ii) the expressiveness of its query language. In order to evaluate these two features, we have proposed six representative queries that cover a wide range of Grid systems, including Grid hardware resources, software resources, middleware environment, services, applications, etc., and show increasing complexity. These queries can be normally issued by middleware systems like schedulers, resource brokers or by more complex applications:

- Query 1: Find all the Computing Elements (CEs) that support the BIOMED Virtual Organisation (VO).

- Query 2: Find all the CEs that support the BIOMED VO and have more than 100 CPUs available.

- Query 3: Find all the CEs that support the MPI running environment.

- Query 4: Find all the CEs that support the BIOMED VO, have more than 100 CPUs available, and support the MPI running environment.

\footnotetext{
${ }^{1}$ ui.tier2.hep.manchester.ac.uk

2 ce.phy.bg.ac.yu
} 
- Query 5: Find all the CEs where GATE (Geant4 Application for Tomographic Emission) can be run.

- Query 6: Find all the CEs that support the BIOMED VO, have more than 100 CPUs available, and where GATE can be run.

Table 1. An Example of the Query 1 in BDII, RGMA, and ActOn

\begin{tabular}{|c|c|}
\hline $\begin{array}{l}\text { Information } \\
\text { Service }\end{array}$ & Query 1 \\
\hline $\begin{array}{l}\text { BDII } \\
\text { (LDAP } \\
\text { Search) }\end{array}$ & $\begin{array}{l}\text { ldapsearch -x -H ldap://lcg-bdii.cern.ch: } 2170 \\
\text {-b mds-vo-name=local,o=grid '( } \& \text { (objectClass=GlueVOView) } \\
\text { (GlueVOViewLocalID=biomed))' GlueCEAccessControlBaseRule }\end{array}$ \\
\hline $\begin{array}{l}\text { RGMA } \\
\text { (SQL Query) }\end{array}$ & $\begin{array}{l}\text { select GlueCEVOViewUniqueID, Value from } \\
\text { GlueCEVOViewAccessControlBaseRule WHERE Value='VO:biomed' }\end{array}$ \\
\hline $\begin{array}{l}\text { ActOn } \\
\text { (SPARQL } \\
\text { Query) }\end{array}$ & $\begin{array}{l}\text { PREFIX egeeOnto: <http://www.cs.man.ac.uk/img/ontogrid\#> } \\
\text { SELECT ?ceid ?ceID ?VO } \\
\text { WHERE } \\
\text { ?ceid egeeOnto:CEUniqueID ?ceID. } \\
\text { ?ceid egeeOnto:hasVO ?VO . } \\
\text { OPTIONAL \{ ?ceid egeeOnto:VO ?ceID. } \\
\text { FILTER ( ?vo = '(biomed')') }\end{array}$ \\
\hline
\end{tabular}

Each of these six queries has been translated into the query languages of the three information services. Table 1 shows an example for Query 1 . And we use different clients to execute these queries and extract the results obtained (e.g., ldapsearch for BDII, the gLite RGMA client tools for RGMA and a Java-based ActOn client for the ActOn-based information service.

Not only queries are different, but also query results are obtained in different manners, due to the differences in the information models of each service. The result of a BDII query is a set of LDAP entries, of an RGMA query a set of table rows, and of an ActOn-based query a set of RDF triples. Figure 4 shows three different ways to show the same Grid resource in the three services evaluated (i.e., ce02.tier2.hep.manchester.ac.uk, an EGEE Computing Element). Even if they have different syntax and size, in our experiment we count them as one piece of information each. That is, we use each "Grid resource" obtained from a query as the basic unit for counting information, which will be used to calculate precision and recall, as described in Section 3.3.

\subsection{Experimental Results Measurement}

The experiment consists in examining the information retrieved for each of the six queries aforementioned, so as to get their corresponding precision and recall measures.

Precision is easy to determine, since it can be computed manually by looking at the results obtained from each query. In all cases, we assume binary relevancy of information, that is, each piece of information retrieved is either relevant or irrelevant for the issued query.

Recall is more difficult to determine, due to the fact that the amount of information available in the EGEE production testbed changes frequently in these systems and there is no way to 

Query results of BDII:
\# biomed, ce02.tier2.hep.manchester.ac.uk:2119/jobmanager-Icgpbs-biomed, UKI-NORTHGRID-MAN-HEP, local, grid
dn: GlueVOViewLocalID=biomed,GlueCEUniquelD=ce02.tier2.hep.manchester.ac.uk:2119/jobmanager-Icgpbs-
biomed,mds-vo-name=UKI-NORTHGRID-MAN-HEP,mds-vo-name=local,o=grid biomed, mds-vo-name=UKI-NORTHGRID-MAN
GlueCEAccessControlBaseRule: VO:biomed

Query results of RGMA:

\begin{tabular}{|c|c|}
\hline | GlueCEVOViewUniqueID & I Value \\
\hline
\end{tabular}

|ce02.tier2.hep.manchester.ac.uk :2119/jobmanager-Icgpbs-biomed/biomed | Vo:biomed |

Query results of ActOn:

| ceid
| <http://img.cs.man.ac.uk/ontogrid1234423456> | | "celD

Figure 4. Results of BDII, RGMA, and ActOn for the the same Grid resource Computing Element at University of Manchester (ce02.manchester.ac.uk)

get accurate information about the actual state of the Grid resources that are available without using the information services that we are evaluating. To get a good approximation that can be used for our purposes, we execute each query 100 times, with a 4-minute interval between executions, that is, we monitor the testbed during 400 minutes. Then we use the highest value obtained from this 100 executions as the total number of relevant information to be used to calculate recall.

\section{Evaluation Results and Conclusions}

Tables 2, 3 and 4 provide the precision and recall measurements obtained after the execution of the experiments described above for the three information services selected: BDII, RGMA and the ActOn-based information service. The values provided in the tables show the average of executing the queries 100 times.

Table 2. BDII Recall \& Precision Measurement (100 times)

\begin{tabular}{|c|cc|c|r|}
\hline QueryNo. & Retrieved Info. & Relevant Info. & Precision & Recall \\
\hline 1 & 14,999 & 15,200 & 1 & 0.987 \\
2 & 242,517 & 19,708 & 0.082 & 0.918 \\
3 & 7174 & 7300 & 1 & 0.983 \\
4 & 485034 & 4600 & 0.010 & 0.990 \\
5 & - & - & - & - \\
6 & - & - & - & - \\
\hline
\end{tabular}

Table 3. RGMA Recall \& Precision Measurement (100 times)

\begin{tabular}{|c|cc|c|r|}
\hline QueryNo. & Retrieved Info. & Relevant Info. & Precision & Recall \\
\hline 1 & 3417 & 15200 & 1 & 0.225 \\
2 & 6321 & 6321 & 1 & 1 \\
3 & 6568 & 7300 & 1 & 0.900 \\
4 & 11245 & 4914 & 0.437 & 0.563 \\
5 & - & - & - & - \\
6 & - & - & - & - \\
\hline
\end{tabular}

As a general comment about these results, we can highlight the fact that BDII shows in general poor results with respect to recall and precision, while ActOn and RGMA present better results. This is mainly related to the repository that BDII uses (LDAP), which is too lightweight and 
Table 4. ActOn Recall \& Precision Measurement (100 times)

\begin{tabular}{|c|cc|c|r|}
\hline QueryNo. & Retrieved Info. & Relevant Info. & Precision & Recall \\
\hline 1 & 15200 & 15200 & 1 & 1 \\
2 & 34100 & 34100 & 1 & 1 \\
3 & 6568 & 7300 & 1 & 0.900 \\
4 & 6568 & 7300 & 1 & 0.900 \\
5 & 24 & 24 & 1 & 0.900 \\
6 & 6 & 6 & 1 & 1 \\
\hline
\end{tabular}

hence provides weak information process and query capabilities; while RGMA's is based on relational databases and ActOn's is based on RDF, which both have better query capabilities.

Now we will analyse with more detail some of the system behaviours over specific queries, and derive more conclusions from these values:

BDII has weak query capabilities. Table 2 shows that BDII has extremely bad precision results for queries 2 and 4, while the results for queries 1 and 3 are excellent. This is related to its weak query ability, as aforementioned. LDAP-based queries are string-based, and hence they cannot be used to support queries over numerical values, such as "greater than or lower than". If we want to improve this precision value, we need to fetch all the information about CE CPUs as a string value first (as we have done to get these results), and then post-process (filter) those results on the client side. RGMA and the ActOn-based information services do not have that problem, since their query abilities are better.

RGMA is not able to relate information available in different tables. Table 3 shows that RGMA has bad precision results in query 4. RGMA contains information to solve this query, but the information comes from two different tables (GlueCE and GlueSubClusterSof twareRunTimeEnvironment), and the query language used by RGMA does not allow making a join of both tables. Hence the situation is similar to the previous case: this problem can be solve on the client side by post-processing the results that have been obtained from each separate query.

RGMA is very sensitive to the registering and availability of information providers at a given point in time. Table 3 shows that RGMA has bad recall results in query 1 . This is because the amount of Computing Element producers that is available during the experiment is not always stable, due to the fact that either producers were not registered in the RGMA registry at that specific moment, or that the producers were not configured correctly or available at that point in time. BDII and the ActOn-based information service are more robust to this, due to the fact that they store information locally and do not depend on their information providers at the time of querying.

Some complex queries cannot be answered by one type of information service in isolation. Tables 2 and 3 show that BDII and RGMA can only answer the first four queries. They cannot answer queries 5 and 6 because their information providers cannot provide enough information and should be combined. This shows that the ability of BDII and RGMA to share their data resources is weak. On the other hand, the ActOn-based information service has the ability to adopt existing information sources as its information providers, and aggregate information from these information sources to answer such complex queries.

\section{Lessons learned}

The experience of developing the experiments for information quality measurement and conducting them on the EGEE Grid testbed has generated several valuable lessons, most of them related to the fairness of the information quality measurement process, which can be applicable to other similar types of experiments.

First, it is difficult to find standard domain-independent methods to measure information quality in information systems. Hence if we want to design and run an experiment in 
a specific domain (e.g., Grid information services), we must design it according to that domain and the information needs of the information service users (either other applications or end-users).

Second, different information services use different information models, and usually provide different expressivity in their query languages or access APIs. This means that a special effort has to be made in order to define clearly a fair way to perform measurements that takes into account these differences.

We think that the results that we have presented can be of great help for the developers who work in the implementation of these and other similar information services, so that they can use these experiments as a benchmark suite, and for the developers of information-intensive applications that make use of these services. In [13], there is also data available about the performance of the three information services for the same set of queries. We do not include a discussion about those experiments since they are out of the scope of this paper, but the main summary would be that BDII and the ActOn-based information service are similar with respect to their response time and RGMA is generally slower than them, due to its information management architecture.

\section{Acknowledgements}

This work is supported by the EU FP6 OntoGrid project (STREP 511513) funded under the Grid-based Systems for solving complex problems, by the Marie Curie fellowship RSSGRID (FP6-2002-Mobility-5-006668), and by the EU FP6 CoreGrid Network of Excellence (FP6-004265). We also thank Pinar Alper (IMG group), Antun Balaz and Laurence Field (EGEE porject), Georges Da Costa and Anastasios Gounaris (CoreGrid WP2), for their helpful comments. 


\section{References}

[1] X. Zhang, J. Freschl, and J. Schopf., "A performance study of monitoring and information services for distributed systems," in 12th International Symposium on High-Performance Distributed Computing (HPDC12 2003). Seattle, WA, USA: IEEE Computer Society, 2003.

[2] X. Zhang and J. Schopf, "Performance analysis of the globus toolkit monitoring and discovery service, mds2." in the International Workshop on Middleware Performance (MP 2004), part of the 23rd International Performance Computing and Communications Workshop (IPCCC), April 2004.

[3] K. Czajkowski, S. Fitzgerald, I. Foster, and C. Kesselman, "Grid information services for distributed resource sharing," in Proceedings of the Tenth IEEE International Symposium on High-Performance Distributed Computing (HPDC-10). IEEE Press, August 2001.

[4] "Berkeley Database Information Index (BDII)," http://lfield.home.cern.ch/lfield/cgibin/wiki.cgi?area=bdiipage $=$ documentation.

[5] E. W. Team, "EDG RGMA,” www.marianne.in2p3.fr/datagrid/documentation/rgma-guide.pdf.

[6] “European DataGrid,” http://eu-datagrid.web.cern.ch/eu-datagrid/.

[7] J. Marco and et al., "First Prototype of the Crossgrid Testbed," in Proceedings of First European AcrossGrids Conference (AXGrids 2003), LNCS 2970. Santiago de Compostela, Spain: Springer-Verlag, 2003, pp. 67-77.

[8] “Enabling Grids for E-sciencE (EGEE),” http://public.eu-egee.org/.

[9] “Globus Toolkit," http://www.globus.org/toolkit/.

[10] "The Spallation Neutron Source (SNS) project," http://www.sns.gov/.

[11] W. Xing, O. Corcho, C. Goble, and M. Dikaiakos, "A Grid Information Service based on an Intelligent Information Integration Architecture," in Europe Semantic Web Conference 2007 (ESWC-2007), 2007, Poster.

[12] M. Parkin, S. van den Burghe, O. Corcho, D. Snelling, and J. Brooke, "The Knowledge of the Grid: A Grid Ontology," in Proceedings of the 6th Cracow Grid Workshop, Cracow, Poland, October 2006.

[13] “OntoGrid CVS,” http://www.ontogrid.net/ontogrid/downloads.jsp.

[14] R. Wang and D. Strong, "Beyond Accuracy: What Data Quality Means to Data Consumers," Management Information Systems, vol. 12, no. 4, pp. 5-34, 1996.

[15] N. Dushay and D. Hillman, "Analyzing metadata for effective use and re-use." in the international DCMI Metadata Conference and Workshop., 2003.

[16] B. Hughes, "Metadata quality evaluation: Experience from the open language archives community." in ICADL, 2004, pp. 320-329.

[17] I. Foster, C. Kesselman, G. Tsudik, and S. Tuecke, "A Security Architecture for Computational Grids." 5th ACM Conference on Computer and Communications Security Conference, 1998.

[18] “OntoGrid CVS at UoM,” http://rpc314.cs.man.ac.uk. 